\title{
Gemeinsamkeiten der allergischen Rhinitis und des allergischen Asthma bronchiale? Die „One-Airway-One-Disease“-Hypothese
}

Teil 1: Epidemiologie und Pathophysiologie

\author{
A Connection Between Allergic Rhinitis and Allergic Asthma? The "One-Airway- \\ One-Disease"-Hypothesis. Part 1: Epidemiology and Pathophysiology
}

\begin{abstract}
A. Gillissen ${ }^{1}$
G. Höffken²

U. R. Juergens ${ }^{3}$
\end{abstract}

\section{Zusammenfassung}

Anatomische und physiologische Besonderheiten und die Trennung durch die betroffenen, medizinischen Fachgebiete haben zu einer gesonderten Betrachtungsweise zwischen den oberen und den unteren Atemwegen geführt. In Bezug auf die allergische Rhinitis und des allergischen Asthma bronchiale muss die Richtigkeit einer solchen Trennung aber aus folgenden Gründen angezweifelt werden: Ähnliche bzw. identische und miteinander gekoppelte immunologische Entzündungsabläufe und -reaktionen, die auf den Haupteffektorzellen, nämlich den Mastzellen und eosinophilen Granulozyten beruhen. Epidemiologisch häufig gemeinsames Vorkommen beider Erkrankungen sowie genetische Gemeinsamkeiten. Ob es sich bei der Rhinitis und dem Asthma in Wirklichkeit lediglich um eine einzige allergische Grundkrankheit mit unterschiedlichen Ausprägungsgraden handelt, wird in diesem ersten Teil diskutiert.

\section{Abstract}

The nose and the lungs are anatomically and physiologically divided which lead to separated strategies in diagnostic and therapy. The upper airways, from the nose and lungs may account for the traditional division in upper and lower airways. Nonetheless a link between upper and lower respiratory tracts has been repeatedly observed in the past decades making the current division in two separate entities an arbitrary dichotomy. Once allergic rhinitis and asthma are two manifestation of the atopic syndrome it is logical to expect that allergy is not a disease confined to specific target organ rather to a broad spectrum of clinical manifestations. This hypothesis has been supported from various observations: Both, allergic asthma and allergic rhinitis are characterized by a similar if not an identical inflammatory process in which mast cells and eosinophils appear to be the major effector cells, high comorbidity of both allergic manifestations as shown in epidemiologic studies. Both diseases are caused by the interaction of genetic susceptibility with environmental factors. In this review, the latest developments in epidemiology and pathophysiology with regard to nasobronchial interaction in allergic airway disease will be discussed. 


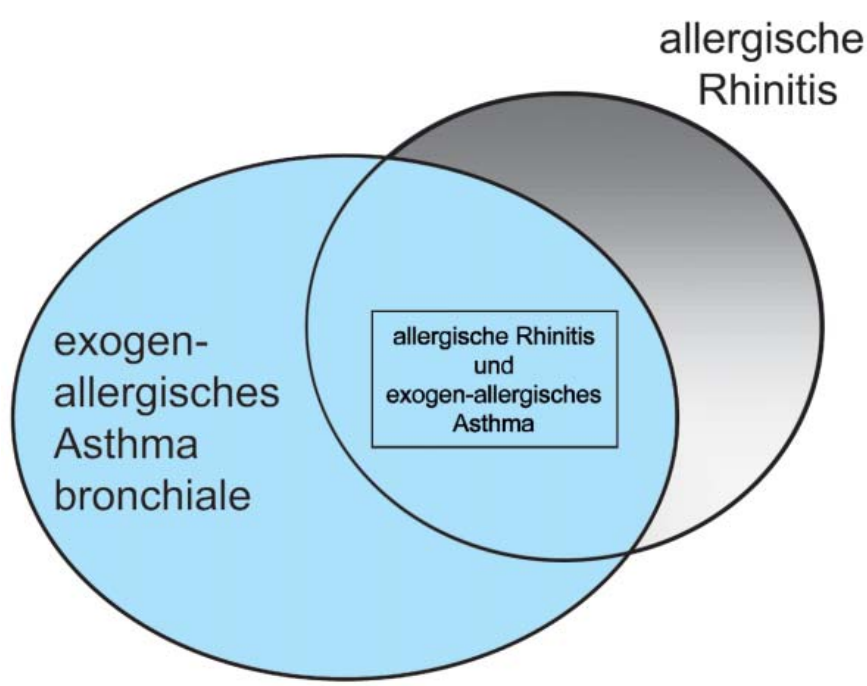

Abb. 1 Die Rhinitis allergica und das exogen-allergische Asthma bronchiale haben viele immunologische, klinische und therapeutische Gemeinsamkeiten.

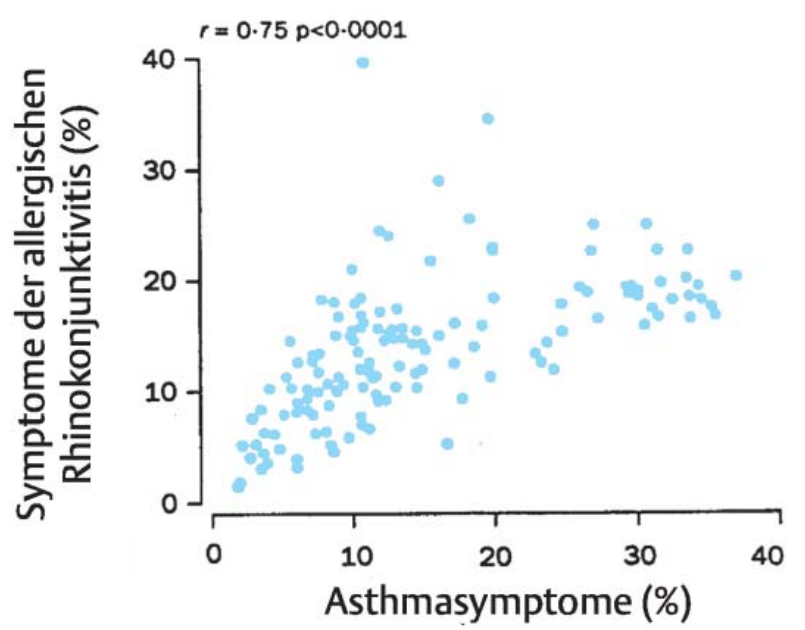

Abb. 2 Zusammenhang zwischen den Symptomen einer Rhinitis allergica und einem Asthma bronchiale bei Kindern in der ISAAC-Studie (International Study of asthma and Allergies in Childhood) [2]. SIT = Spezifische Immuntherapie.

Rhinokonjunktivitis und des Asthma bronchiale mit Betonung der industrialisierten Staaten: Während der letzten zwei Dekaden stieg die Asthmaprävalenz z. B. in Europa um ca. das Doppelte, die der Rhinitis allergica um ca. das Dreifache an [6]. Zwischen 1968 und 1989 verdoppelte sich in Australien die Prävalenz der allergischen Rhinitis von ca. $20 \%$ auf ca. $40 \%$. In Finnland stieg zwischen 1977 und 1991 die Prävalenz der Rhinitis von 5\% auf $14,9 \%$ und die des Asthmas von 1 auf 2,9\% an [7]. Weitere Hinweise auf die enge Verknüpfung beider Erkrankungen ergeben sich aus folgenden epidemiologischen Beobachtungen:

- Kinder mit einer Rhinitis allergica haben ein ca. dreifach erhöhtes Risiko im Erwachsenenalter ein exogen-allergisches Asthma bronchiale zu entwickeln $[8,9]$.

- Beide Erkrankungen haben einen gemeinsamen genetischen Hintergrund. So ist das Erkrankungsrisiko für ein Asthma bei Kindern atopischer gegenüber Kindern nicht-atopischer Eltern erhöht $[8,10,11]$. 
- In epidemiologischen Studien wurde bis zu 80\% aller Asthmapatienten auch eine Rhinitis allergica gefunden [7,12]. Allerdings schwanken diese Angaben in Abhängigkeit vom Rekrutierungsdesign und der Allergiedefinition. In der Copenhagen Allergy Study wiesen $41 \%$ der Pollen-sensibilisierten Patienten auch ein pollensensitives Asthma bronchiale auf [13]. Umgekehrt war ein allergisches Asthma fast immer auch mit einer Pollen-Rhinitis assoziiert. Nur $0,1 \%$ aller Asthmapatienten hatten keine Rhinitis. Für andere Allergene ergaben sich folgende Verhältnisse: Tierhaarallergie ( $52 \%$ und $0,2 \%$ ), Hausstaubmilbenallergie ( $41 \%$ zu $1 \%$ ). Daraus errechnete sich bei Rhinitis-Patienten gegenüber nicht-allergischen Personen ein 300-fach erhöhtes Risiko ein Asthma zu entwickeln $[13,14]$.

- Beide Erkrankungen haben wesentliche immunologische Gemeinsamkeiten (Zytokinmuster, zelluläres Entzündungsbild in der jeweiligen Schleimhautbiopsie).

Beim European Community Respiratory Health Survey wurden nach einem Zufallsprinzip 20 - 44-Jährige zu Allergiesymptomen und Asthma befragt. Die Studienteilnehmer mit einer perennialen Rhinitis gaben im Vergleich zu den gesunden Kontrollen häufiger an, an einem Asthma zu leiden. Selbst unter Berücksichtigung von Geschlecht, Alter, Rauchgewohnheiten und familiärer Disposition war das exogen-allergische Asthma streng (odds ratio 8,1; 95\% Konfidenzintervall 5,4-12,1) mit der allergischen Rhinitis assoziiert [12]. In einer mittels Pricktest und einer Befragung an 977 australischen Olympia-Athleten durchgeführten Untersuchung zeigte sich, dass $37 \%$ an einer Allergie und unter diesen $36 \%$ zusätzlich an einem Asthma litten. 37\% der Asthmatiker wiesen eine saisonale Allergie auf $[15,16]$.

\section{Allergie als Risikofaktor für die Entwicklung eines Asthma bronchiale}

Die Allergie ist für die Entwicklung eines allergischen Asthma bronchiale ein unabhängiger Risikofaktor, wie epidemiologische und Therapiestudien belegen konnten [12]. In einer schwedischen Studie hatten Erwachsene mit einer atopischen Familienanamnese (Asthma und/oder Rhinitis bei Familienmitgliedern) ein 3- bis 4-fach erhöhtes Risiko, ein Asthma zu entwickeln und ein 2- bis 6-fach erhöhtes Risiko an einer Rhinitis zu erkranken als familiär Unbelastete [17]. In der Copenhagen Allergy Study wurden 734 symptomatische Patienten, die aus einer Bevölkerungsbefragung mit insgesamt 8000 versandten Fragebogen ausgewählt wurden, 1990 sowie acht Jahre später untersucht. 26,0\% der Rhinitis-Patienten wiesen schon bei Studienbeginn ein Asthma auf. Bei Studienende stieg dieser Prozentsatz auf 28,4\%. 14,1\% der Patienten mit einer Pollensensibilisierung (initial kein Asthma) entwickelten innerhalb dieser acht Jahre ein Pollen-sensitives Asthma. Im Gegensatz dazu erkrankten daran lediglich 1,8\% der bei Studienbeginn nicht gegenüber Pollen sensibilisierten Patienten. Ähnlich stellt sich die Asthmainzidenz bei Tierhaarsensibilisierten (16,0\%; $0,9 \%$ bei fehlender Sensibilisierung) und deutlicher sogar bei Hausstaub-sensibilisierten Patienten dar $(26,3 \%$ vs. $0,7 \%)[14]$.

Die Häufigkeit des bei der Diagnosestellung gemeinsamen Vorkommens einer allergischen Rhinitis und einem allergischen Asthma ist vom Patientenalter abhängig. Yawn u. Mitarb. fanden bei knapp 60\% der untersuchten Asthmapatienten (Diagnose- stellung bis zum 25. Lebensjahr) eine gleichzeitig bestehende Rhinitis. Wird die Asthmadiagnose jedoch erst jenseits des 40 . Lebensjahrs gestellt, haben nur noch $15 \%$ der Patienten eine solche Koexistenz beider Erkrankungen [18]. Andere Studien bestätigten diese Beobachtung $[2,19,20]$.

In einer Studie über 10 Jahre entwickelten von 154 Kindern (Alter 3 - 17) mit allergischer Rhinitis 19\% ein Asthma. Die Asthmaprävalenz war bei Kindern mit einer perennialen Rhinitis doppelt so hoch ( 15 von $44 ; \mathrm{p}<0,01$ ) als bei denjenigen mit einer saisonalen Rhinitis (14 von 110) [21]. Eine solche Konstellation ist häufiger mit schweren Krankheitsverläufen und mit häufigeren bakteriellen und viralen Infektionen assoziiert [22]. Die Behandlungskosten liegen bei Kindern und Erwachsenen, die an beiden Erkrankungen leiden, mit $7928 € / J a h r$ (Kinder) bzw. $9287 € / J a h r$ (Erwachsene) deutlich höher, als bei denjenigen Asthmapatienten, die keine begleitende atopische Genese aufweisen (Kinder 1089 $€ / J a h r$; Erwachsene $1543 € / J a h r$ ) [23].

Upton u. Mitarb. publizierten eine 20-Jahres-Verlaufsstudie, in der die Bevölkerung zur Prävalenz von Asthma, Heuschnupfen und pulmonalen Symptomen befragt wurde. Es wurden die Angaben der Jahre 1972-76 mit denen von 1996 verglichen. Danach stieg bei Nichtrauchern die Heuschnupfen-Prävalenz von $5,8 \%$ auf $19,9 \%$ und auch die des Asthmas von 3,0\% auf $8,2 \%$ an. Bei Rauchern ergaben sich folgende Anstiege: Heuschnupfen von 5,3\% auf $15,5 \%$ und Asthma von 1,6\% auf 5,4\% [6]. In einer weiteren, über 22 Jahre durchgeführten Untersuchung wurde dieser Trend bestätigt. In Poole/England wurden 100 Säuglinge, deren Mutter und/oder Vater eine Atopie aufwiesen, ab dem Vorschulalter jährlich und danach im 11. und 22. Lebensjahr untersucht. Es wurden jedes Mal ein Prick-Test mit Standard-Allergenen sowie eine IgE-Bestimmung im Blut durchgeführt. Darüber hinaus wurden im 11. sowie im 22. Lebensjahr die bronchiale Hyperreaktivität (BHR) mittels Histamin quantifiziert. Die Typ-I-Sensibilisierungen gegenüber Aeroallergenen stiegen bis Studienende auf 70\% der Gesamtkohorte bzw. allein bei den Asthmapatienten auf $60 \%$ an. Die BHR-Ergebnisse der 22-Jährigen waren von dem Beginn der Asthmasymptomatik abhängig. BHR-positiv waren am Studienende: $16 \%$ der Untersuchten, die niemals über Giemen geklagt hatten, $8 \%$ derjenigen, die nur im Alter $<5$ Jahre, aber $75 \%$, die im Alter von $>5$ Jahren giemten [24].

Als ein weiterer epidemiologischer Hinweis auf die enge pathophysiologische Beziehung zwischen Allergie und Asthma scheint die Sensibilisierung gegenüber Tierallergenen (>12-monatige Haustierhaltung: Katzen, Hunde, Nager, Vögel) die Asthmainzidenz (odds ratio 1,39; $95 \%$ Konfidenzintervall 1,05 - 1,84) zu fördern, wie in einer in Südfinnland durchgeführten Populationsstudie an neu diagnostizierten Asthmapatienten gezeigt werden konnte. Die höchste Wahrscheinlichkeit, ein Asthma zu entwickeln errechnete sich bei Patienten mit atopischen Eltern und einer gleichzeitig bestehenden Haustierhaltung (odds ratio 2,39, 1,55-3,69). Bei diesen Patienten war das Asthmarisiko im Vergleich zur gesunden Vergleichsgruppe um 39\% erhöht [25]. 
Bronchiale und nasale Hyperreaktivität bei Atopie ein Bindeglied zwischen Allergie und Asthma?

Wenn ein ätiologischer Zusammenhang zwischen Asthma und allergischen Erkrankungen der oberen Atemwege besteht, so muss auch ein Zusammenhang zwischen BHR und Atopie und/ oder Asthma beobachtbar sein. Die BHR, definiert als eine im Vergleich zum Lungengesunden gesteigerte Reaktion der Atemwege nach Stimulation mit physikalischen, chemischen oder pharmakologischen Reizen, ist Bestandteil der Asthmadefinition. Eine antiinflammatorische Therapie kann eine BHR abschwächen $[26,27]$.

Die BHR kommt in Studien bei ca. 20\% asymptomatischer Probanden vor, ist häufiger bei Patienten mit einer Atopie mit oder ohne einem begleitendem Asthma und wird als Folge einer bestehenden bronchialen Inflammation angesehen, die zu Asthma führen kann oder schon Ausdruck eines frühen, klinisch noch nicht erkennbaren Krankheitsstadiums ist [28-31]. Das Provokationsergebnis ist allerdings von dem gewählten Provokationsmittel abhängig. Patienten mit einer Atopie reagieren im BHRTest z.B. empfindlicher auf eine Adenosin- als auf eine Metacholin-Exposition [32]. Ebenso weisen atopische Asthmapatienten eine höhere bronchiale Empfindlichkeit auf als diejenigen mit fehlender Sensibilisierung [31]. Die höhere Empfindlichkeit gegenüber Adenosin resultiert wahrscheinlich aus den mastzellaktivierenden und Mediator-freisetzenden (z.B. Histamin, Zytokine) Eigenschaften dieser Substanz [33]. Bei Beschäftigten im Bäckerhandwerk und in der Labortierhaltung $(n=230$, allergische Rhinitis 21,4\%, exogen-allergisches Asthma 10,3\%) wurden unspezifische Provokationstests einerseits mit Methacholin und andererseits mit AMP durchgeführt und die Ergebnisse miteinander verglichen. Die höchste BHR zeigte sich bei Asthma, bei Patienten mit einem positiven Prick-Tests, einer gleichzeitig bestehenden Bluteosinophilie und/oder einer allergischen Rhinitis. Dabei war das BHR-Prävalenzverhältnis in der AMP-Testreihe höher als im Methacholin-Test [32].

In den neuen Bundesländern stieg bei Schulkindern die Inzidenz der mit kalter Luft $\left(-15^{\circ} \mathrm{C}\right)$ gemessenen BHR von $6,4 \%$ $(1992-1993)$ auf $11,6 \%(1995-1996)$ an (odds ratio 1,9, 95\% Konfidenzintervall: 1,3-2,9). Allerdings nahm während dieser beiden Beobachtungszeiträume die Allergiehäufigkeit nur im Trend zu. Auch die Häufigkeit der Diagnose Asthma änderte sich nicht signifikant. Trotzdem vermuten die Autoren, dass die zunehmende BHR einer steigenden Asthmaprävalenz vorausgehen könnte [34]. Von Mutius u. Mitarb. beobachteten dagegen in ihrer vergleichenden Untersuchung in den Jahren 1991 - 1992 bzw. 1995-1996 eine Zunahme der Sensibilisierungshäufigkeit und des Heuschnupfens, nicht jedoch der BHR und des Asthmas $[3,5]$. Trotz dieser nicht ganz einheitlichen Ergebnisse besteht bei vielen Patienten ein Zusammenhang zwischen der BHR einerseits und der allergischen Sensibilisierung bzw. der Entwicklung eines exogen-allergischen Asthmas andererseits [35]. Allerdings ist davon auszugehen, dass dieser Zusammenhang individuell unterschiedlich ausgeprägt ist, wie in einer kanadischen Studie gezeigt werden konnte [28]. Darin wurde die BHR-Entwicklung von 30 gesunden Personen mit einer BHR (Methacholin), mit 30 symptomatischen Asthmapatienten und 30 Gesunden (keine BHR, kein Asthma) über einen Beobachtungszeitraum von 3 Jahren verglichen. Atopische Asthmapatienten wiesen eine höhere BHR auf als Asthmapatienten ohne Atopie: $\mathrm{PC}_{20}$ (Methacholinmenge, die eine $20 \%$ ige Senkung der $\mathrm{FEV}_{1}$ bewirkt) 2,7 \pm 1,1 vs. $4,9+1,1(\mathrm{p}=0,01)$. Unter den BHR-positiven Patienten (mit und ohne Asthma) wiesen $75 \%$ eine Atopie auf. Je schwerer die BHR derjenigen Studienteilnehmer war, die initial als gesund (kein Asthma) klassifiziert wurden, desto wahrscheinlicher wurde die Asthmadiagnose bei Studienende $(p=0,01): 40 \%$ der asymptomatischen Probanden mit einer $\mathrm{PC}_{20}<4 \mathrm{mg} / \mathrm{ml}$ entwickelten ein Asthma. Das gleiche Ergebnis ergab sich bei denjenigen mit einer positiven Familienanamnese für Asthma.

Bei 324 Schulkindern aus Montreal war eine positive Sensibilisierung gegenüber Hausstaubmilben (Prick-Test) der wichtigste unabhängige Risikofaktor für eine BHR $(p<0,001)$. Dagegen führte die Pubertät und die damit verbundene hormonelle Umstellung zu keiner Änderung der bronchialen Empfindlichkeit [36].

\section{Pathophysiologie bei allergischer Rhinitis und allergischem} Asthma

Allergisches Asthma und allergische Erkrankungen der oberen Atemwege haben eine gemeinsame genetische Disposition und besitzen ein gemeinsames immunologisches Entzündungsmuster [37].

\section{Genetische Gemeinsamkeiten}

Die Disposition für eine allergische Erkrankung gilt als gesichert und beruht auf einer komplexen Interaktion verschiedener Gene. Allerdings führt erst die Interaktion mit Umweltfaktoren (Allergene und nicht-spezifische Reize) bei entsprechender genetischen Disposition zur klinischen Manifestation. Bei der Suche nach genetischen Determinanten, die zum Asthma-Phänotyp prädisponieren, wurden verschiedene Wege beschritten, wie z.B. Familien- und Zwillingsstudien, Suche nach Polymorphismen und Änderung von Genloci. Danach wurden von verschiedenen chromosomalen Regionen eine Verbindung zum allergischen Asthma berichtet, so z. B. von den Chromosomen 5q, 6pp, 11q und 13q. Weitere Studien fanden eine Verbindung zu den Chromosomen 1, 2q, 3, 14, 9, 16, 17q sowie in einer Studie zu 7p, 10q, 19, 20 und 21. So wurde die Regulation des bei dieser Population häufig erhöht gefundenen IgE mit dem Chromosom $5 q$ (D5S1480) in Verbindung gebracht. Zudem befinden sich auf 5q31 - 33 die Gene diverser Zytokine, wie z. B. Interleukin (IL)-3, IL-4, IL-5, IL-9, IL-13 und die $\beta$-Kette von IL-12, den granulocytemacrophage colony-stimulation factor (GM-CSF) sowie Gene, die für den $\beta$ - und den Glukokortikosteroidrezeptor kodieren $[38,39]$. Die Chromosom 11q-Region wurde für die Regulation des IgE-Rezeptors Fc\&R bei Patienten mit einem atopischen und gleichzeitig auch dem asthmatischen Phänotyp in Verbindung gebracht. Eine Verbindung von Allergie und Asthma zum Chromosom 11 und dem dort lokalisierten codierten IgE-Locus erbrachte zum ersten Mal die Arbeiten von Cookson u. Mitarb. [40]. Gene des Chromosoms 12q regulieren bei Familien, in denen Atopie und Asthma vermehrt vorkommen, die Expression von Interferon-gamma (IFN- $\gamma$ ), die NO (Stickstoff)-Synthase und dem Mastzell-Wachstumsfaktor. Weitere solcher Genassoziationen wurden auf dem Chromosom 13 gefunden. Bei Allergikern und 
Asthmapatienten wurde zudem über eine Assoziation zu einem IL-4 Rezeptorgenpolymorphismus berichtet [41].

Das Ziel der Multicenter Colaborative Study on the Genetics of Asthma (CSGA) ist die Identifizierung und Lokalisation von Genen, welche die Entwicklung der Phänotypen Asthma, BHR und Atopie fördern bzw. diese Erkrankungen hervorrufen. In dieser Studie sind Gen-Phänotyp-Assoziationen auf den Chromosomen 1p, 2q, 3p, 5q, 6p, 10q, 11q, 12q, 13q, 14q und 16p identifiziert worden. Dort befinden sich Gene, die für die Transkription von Interleukinen, deren Rezeptoren, Wachstums- und Transkriptionsfaktoren, den IgE-Rezeptor, Histokompatibilitätskomplexe u.a. kodieren [41-43].

Studien an Zwillingen und Familienstudien fanden über 12 genomische Regionen, die mit dem Phänotyp Asthma assoziiert sind. Van Eerdewegh u. Mitarb. untersuchten in Genkopplungsanalysen 460 Familien und fanden eine Assoziation zwischen dem Locus auf Chromosom 20p13 einerseits und Asthma und BHR andererseits. Dabei stellte sich bei 135 Polymorphismen in 23 Genen beim ADAM33-Gene eine solche signifikante $(\mathrm{p}<0,04)$ Assoziation heraus [44]. Die ADAM-Genfamilie ist eine Subgruppe der Zink-abhängigen Metalloproteinase-Superfamilie. ADAMProteine bestehen aus insgesamt acht Domeinen (u. a. für Metalloproteinasen, Disintegrin und dem epidermalen Wachstumsfaktor [EGF]). Sie sind entweder in der Zellmembran oder im Golgi-Apparat verankert. Wegen der komplexen Proteinstruktur sind ADAM-Proteine in diversen biologischen Reaktionen involviert, wie der Zellproliferation, Zelldifferenzierung und -migration sowie der Embriogenese via Interaktion mit Integrinen und extrazellulären Matrixproteinen [45]. Eine ähnliche Assoziation wurde aktuell auch zwischen der allergischen Rhinitis gegenüber Pollen des japanischen Zedernbaumes und ADAM33-Genpolymorphismen beschrieben [46]. Die Bedeutung des Zusammenhangs zwischen dem ADAM33 Gen-Polymorphismus und dem Asthma ist allerdings in ihrer Wertigkeit unklar. So wurde kürzlich ein solcher Zusammenhang zwar bei asthmatischen Kindern von insgesamt 652 Familien bestätigt, aber es ergab sich in dieser Untersuchung nur eine marginale Assoziation. Möglicherweise gilt die Assoziation zwischen ADAM33-Gen-Polymorphismen und allergische Erkrankungen nicht uneingeschränkt. So ergaben sich deutliche Unterschiede zwischen den geno- und phänotypischen Ergebnissen zwischen der weißen Bevölkerung bzw. denjenigen spanischer und afrikanischer Abstammung [47].

Von aus dem Blut von Patienten mit einem Asthma und einer atopischen Dermatitis isolierte T-Zellen, die ex vivo mit Dermatophagoides farinae Antigen exponiert wurden, produzieren signifikant höhere IL-5 und IL-13-Mengen als die Zellen gesunder Kontrollen [48]. Zentraler Regulator scheint auf genetischer Ebene die Allergen-induzierte Expression der STAT-Familie zu sein (Signal Transducer and Activator of Transcription). Untersuchungen an verschiedenen Zellkulturen belegen, dass die STAT-1, STAT-5 und STAT-6-Aktivierung einer gesteigerten Expression der Interleukine IL-4 und IL-13 vorgeschaltet ist, so dass STAT diesen Signalweg zu regulieren scheint [49-51]. Da u.a. IL-13 eosinophile Granulozyten aktiviert, ergibt sich über die STAT-Regulation eine weitere genetische Gemeinsamkeit allergischer Erkrankungen, zumal IL-13 und IL-4 auch umgekehrt im Rahmen eines negativen feed-back Mechanismus zu einer Expressionshemmung der IL-13-Rezeptor-alpha 1-Subgruppe auf Zelloberfläche dieser Zellen führen und damit eine Regulatorfunktion besitzen [48,52]. Ein solcher negativer feed-back Mechanismus wurde auch für IL-5 und den IL-5 $\alpha$-Rezeptor beschrieben [53].

Laitinen u. Mitarb. fanden auf Chromosom 7p einen 133-kb (Kilobasen) großen Bereich (Locus), der zwei Gene enthält. Eins dieser Gene codiert für den GPRA-Rezeptor (G protein-coupled receptor for asthma susceptibility), der mit hohen Serum IgE-Spiegeln und dem Asthma-Pänotyp assoziiert waren [54]. Der zu diesem Rezeptor passende Ligand ist allerdings unbekannt. Somit kommt neben anderen Kandidatengene auch die für GPRA codierende Region als genetische Basis für die Entstehung einer Atopie und/oder eines Asthmas infrage.

Tab. 1 gibt die bisherigen Kandidaten-Gene, die mit dem Asthma, der BHR und der Atopie in Verbindung gebracht wurden, sowie ihre chromosomale Lokalisation wieder.

Tab. 1 Kandidaten-Gene für den Phänotyp Asthma bronchiale Hyperreaktivität und Allegie (modifiziert nach [38,44, 49-54]). IL= Interleukin, GM-CSF = granulozcyte-macrophage colony-stimulation factor, IgE =Immunglobulin E; $\mathrm{HLA}=$ human leucocye antigen, TNF- $\alpha=$ Tumor-NekroseFaktor alpha, TcR = T-Zell-Antigenrezeptor, INF- $\gamma=$ Interferon gamma, NFYB $=$ B subunit nuclear factor- $\gamma$, MGF = mast cell growth factor, iNOS = induzierbare Stickoxid-Synthase, STAT = signal transducer and activator of transcription, RANTES = regulated on activation, normal T-cell expressed and secreted. GPRA $=G$ protein-coupled receptor for asthma susceptibility.

\begin{tabular}{|c|c|c|}
\hline $\begin{array}{l}\text { Kandidaten- } \\
\text { Gen }\end{array}$ & Funktion & $\begin{array}{l}\text { Chromosomen- } \\
\text { Region }\end{array}$ \\
\hline IL-1 $\alpha$, IL-1 $\beta$ & Antigenpräsentation & $2 q 21$ \\
\hline $\begin{array}{l}\text { IL-3, IL-4, IL-5, } \\
\text { IL-9, IL-13, } \\
\text { GM-CSF }\end{array}$ & $\begin{array}{l}\text { IgE-regulierende Zytokine, Mastzellen-, } \\
\text { Eosinophilen-Funktion }\end{array}$ & $5 q 31$ \\
\hline $\begin{array}{l}\beta \text {-adrenerger } \\
\text { Rezeptor }\end{array}$ & Rezeptorkinetik & $5 q 32$ \\
\hline HLA-Gen & Antigenpräsentation & $6 p$ \\
\hline TNF- $\alpha$ & Pro-inflammatorisches Zytokin & $6 p 21.3$ \\
\hline TCR $\beta$-Rezeptor & T-Zellaktivierung & 7 \\
\hline FCERI & $\begin{array}{l}\text { Aktivierung von Mastzellen, basophile } \\
\text { Zellen, antigenpräsentierende Zellen } \\
\text { (Dendritische Zellen) }\end{array}$ & $11 q 13$ \\
\hline FCERII & IgE-abhängige Antigenpräsentation & $11 q 13$ \\
\hline $\begin{array}{l}\text { IFN- } \gamma, \text { NYFB, } \\
\text { MGF, iNOS }\end{array}$ & $\begin{array}{l}\text { T-Zellregulation, pro-inflammatorische } \\
\text { Mediatoren }\end{array}$ & $12 q$ \\
\hline $\begin{array}{l}\text { Esterase- } D \\
T C R \\
\alpha-\delta \text {-Komplex }\end{array}$ & T-Zellaktivierung & $\begin{array}{l}13 q \\
14 q 11.2\end{array}$ \\
\hline IL-4-Rezeptor & Zusammen mit IL-4: Induktion von lgE & $\begin{array}{l}16 p \\
17 p 11-q 11.2\end{array}$ \\
\hline $\begin{array}{l}\text { STAT- } 1 /-6 /-5 \text {, } \\
\text { RANTES }\end{array}$ & $\begin{array}{l}\text { Pro-inflammatorische Mediatoren, } \\
\text { Regulation der Interleukinexpression }\end{array}$ & $17 q$ \\
\hline ADAM33 & $\begin{array}{l}\text { Zellproliferation/-differentierung/- } \\
\text { migration, Zelladhäsion, Zellmembran- } \\
\text { fusion, Embriogenese }\end{array}$ & $20 p 13$ \\
\hline GPRA & $\begin{array}{l}\text { Rezeptorprotein, Ligand und Funktion } \\
\text { unbekannt }\end{array}$ & $7 p$ \\
\hline
\end{tabular}




\section{Die immunologische Entzündung}

Im zellulären „Epizentrum“ der allergischen Rhinitis und des Asthma steht die $\mathrm{CD}^{+}{ }^{+}$T-Helfer-Gedächtniszelle. Diese setzt eine Reihe von Zytokinen frei (s.u.), die direkt oder indirekt weitere Entzündungszellen aus der Granulozytenfamilie zur Entzündungsreaktion „programmieren“. Die immunologische Antwort als Reaktion auf eine Allergenexposition ist beim allergischen Asthma und bei der allergischen Rhinitis identisch und im Wesentlichen bekannt. Über Antigen-präsentierende Zellen kommt es beim Allergiker zu einer Aktivierung von $\mathrm{Th}_{2}$-HelferLymphozyten. Diese setzen u.a. IL-4 frei, das für die allergenspezifische IgE-Freisetzung notwendig ist. Weitere im Rahmen der allergischen Reaktion freigesetzte Zytokine sind das IL-13, welches die Entwicklung von Mastzellen und basophilen Granulozyten steuert und Eosinophile aktiviert, und das IL-5, welches zusammen mit IL-3 und dem GM-CSF die eosinophile Entzündungskomponente reguliert. Die Bindung des IgE an spezifische IgE-Rezeptoren auf der Mastzellenoberfläche stimuliert Entzündungszellen zur Freisetzung diverser zellulärer Mediatoren (Leukotriene, Prostaglandine, Eicosanoide, Zytokine, Chemokine, Lipidmediatoren, Enzyme, Wachstumsfaktoren), welche allergische Sofortreaktion auslösen (Abb. 3).

Allergie und Asthma - immunologischen Gemeinsamkeiten Unklar ist derzeit, auf welche Art und Weise die Sensibilisierung zu einer Organmanifestation führt und wie diese „Organselektion“ (z.B. Nase, Haut, Bronchien, Gastro-Intestinaltrakt etc.) auf immunologischer Ebene abläuft. Holt u. Mitarb. schlugen vor, die Entwicklung einer Rhinitis allergica und eines Asthma bronchiale als Zweiphasen-Vorgang zu verstehen [55]. In Phase 1 kommt es mit dem ersten Allergenkontakt zur Ausbildung eines allergen-spezifischen immunologischen Gedächtnisses. Sie umfasst das Säuglings- bis zum Kindesalter, in dem die $\mathrm{Th}_{0} / \mathrm{Th}_{2}$-betonte allergische Sensibilisierungsreaktion stattfindet. Wiederholte Allergenexpositionen führen danach bis zum Jugendalter in der Phase 2 nicht nur zu einer Inflammation in den Schleimhäuten der oberen, sondern zeitverschoben auch der unteren

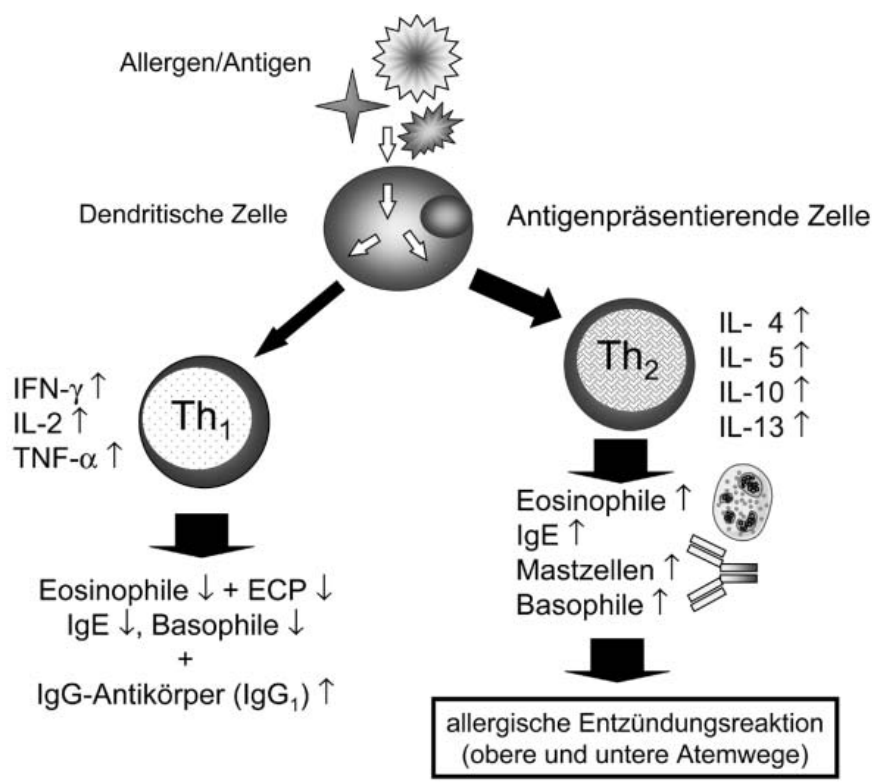

Abb. 3 Ablauf der allergischen Entzündungsreaktion (modifiziert nach [74]).

Atemwege mit Ausbildung eines Asthmas (Abb. 4). Als mögliche Triggerfaktoren werden zusätzlich virale Entzündungen und unspezifische Reize, wie z.B. die Passivrauchexposition von Säuglingen/Kleinkindern rauchender Eltern diskutiert [56 - 58].

Es existieren verschiedene Faktoren, welche die T-Zell-ausgelöste Entzündungsreaktion limitieren helfen. Stickstoffmonoxid (NO) hemmt z. B. die T-Zellaktivierung über T-Zell-Signalkinasen. Da dieser Effekt jedoch ausgeprägter bei $\mathrm{Th}_{1}$ - als $\mathrm{Th}_{2}$-Zellen ist, sind große NO-Mengen für diese antiinflammatorische Wirkung nötig. Die bronchiale NO-Freisetzung ist in den Atemwegen von therapeutisch unzureichend antiinflammatorisch eingestellten oder unbehandelten, klinisch symptomatischen und atopisch reagierenden Asthmapatienten gegenüber Gesunden signifikant

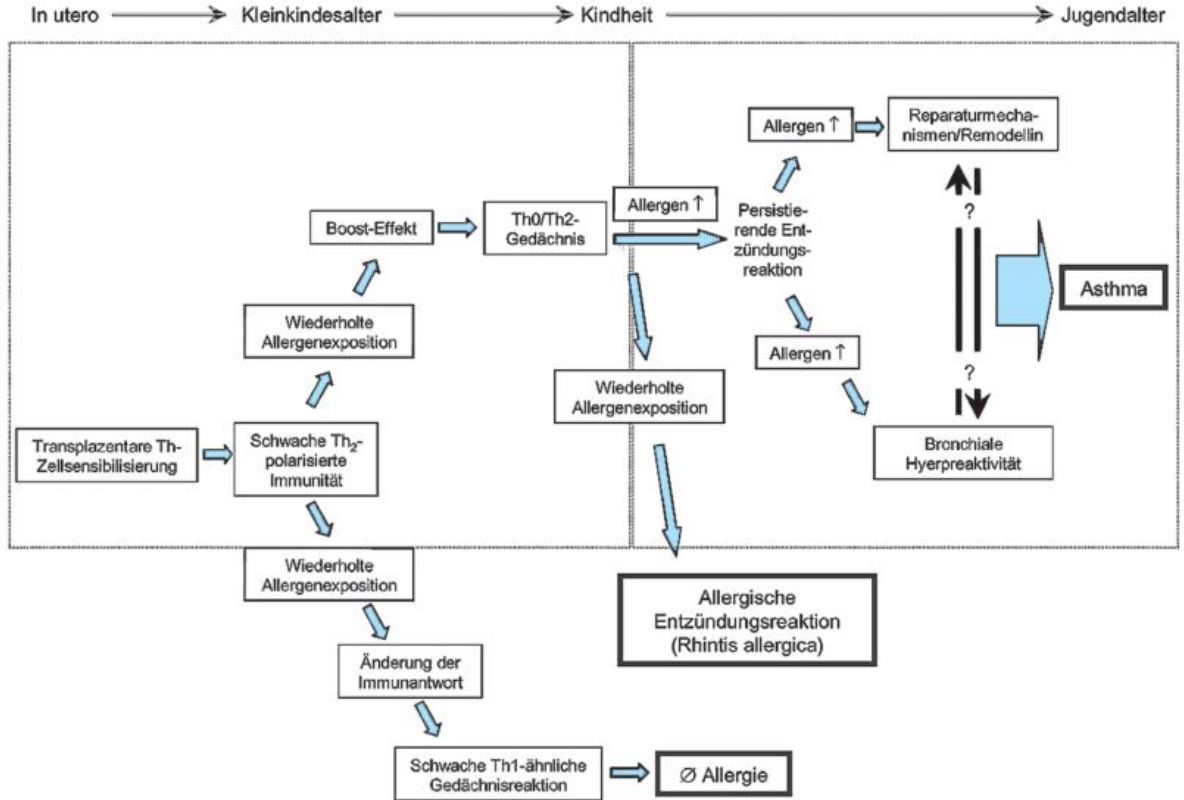

Abb. 4 Verlauf einer frühen allergischen Sensibilisierung im Säuglingsalter. Zeitliche Entwicklung einer Rhinitis und eines exogen-allergischen Asthma bis zum Erwachsenenalter. Dabei geht die allergische Sensibilisierung (mit Rhinitis allergica) der Entwicklung eines Asthmas voraus (modifiziert nach [55]). 
erhöht [59-62]. Zudem hemmen Th${ }_{1}$-Zytokine (IL-2, IFN- $\gamma$, TNF- $\alpha$, Lymphotoxin) die $\mathrm{Th}_{2}$-Antwort. IL-2 und IFN- $\gamma$ unterdrücken durch eine direkte Stimulation der B-Zellen die Synthese des in der immunologischen Reaktion an dominanter Stelle stehende IgE. Einen gleichen Effekt haben IL-12 und IL-18, obwohl sie keine eigentlichen $\mathrm{Th}_{1}$-Zytokine sind.

IgE ist bei allergischen Erkrankungen wichtigstes Bindeglied der immunologischen Entzündung, wie insbesondere bei der atopischen Dermatitis, bei der allergischen Urtikaria, bei der Rhinokonjunktivitis, beim Asthma und bei der anaphylaktischen Reaktion. Die zelluläre IgE-Produktion ist abhängig von $\mathrm{Th}_{2}$-Zytokinen und der $\mathrm{Th}_{2}$-dominierten Entzündungsreaktion mit aktivierten, IL-4 freisetzenden basophilen Granulozyten. Ungeachtet dessen besteht allerdings das derzeit nicht abschließend erklärbare Paradox, dass Patienten mit einer nachgewiesenen atopischen Erkrankung nicht immer erhöhte IgE-Serumspiegel aufweisen, oder dass es anaphylaktoide Reaktionen ohne jegliche IgE-Erhöhungen gibt [63]. Die Bedeutung des IgE bei der atopischen Reaktion wird dadurch allerdings nicht geschmälert. Ganz im Gegenteil belegen die klinischen Erfolge der IgE-Antikörpertherapie bei der allergischen Rhinitis und beim allergischen Asthma die pathogene Bedeutung dieses Immunglobulins $[64,65]$.

Cysteinylleukotriene sind wichtige und ubiquitär vorkommende zelluläre Entzündungsmediatoren beim Asthma und bei der allergischen Rhinitis, die u. a. folgende proinflammatorische Effekte bewirken: Kontraktion der glatten Bronchialmuskulatur, Steigerung des Blutflusses im Gewebe, Förderung der Plasmagewebeexsudation (Ödem), Steigerung der Mukusproduktion glandulärer Zellen, Aktivitätssteigerung granulozytärer Zellen. Leukotriene (LT) entstehen im Arachidonsäurezyklus vor allem in Mastzellen, eosinophilen und basophilen Granulozyten.

$\mathrm{LTC}_{4}, \mathrm{LTB}_{4}$ und $\mathrm{LTD}_{4}$, welche eine vielfach höhere proinflammatorische Wirkstärke als Histamin besitzen, werden sowohl bei der Rhinitis allergica in der nasalen Lavage als auch bei der allergischen Früh- und Spätreaktion des Asthma bronchiale nachgewiesen [66 - 68]. Zudem steigt $\mathrm{LTE}_{4}$, das Endprodukt des Arachidonsäurezyklus sowohl bei der nasalen als auch bei der bronchialen Allergenprovokation an. Umgekehrt lassen sich in beiden Provokationsmodellen die Leukotrien-vermittelte epitheliale Entzündungsreaktion und die daraus resultierende Symptomatik (Fließschnupfen, respektive $\mathrm{FEV}_{1}$-Abfall) durch Leukotrienrezeptorantagonisten (Montelukast, Zafirlukast) hemmen (siehe Therapie, Teil 2) [69-73].

\section{Resümee}

Es gibt starke epidemiologische, genetische und immunologische Hinweise dafür, dass die Rhinitis allergica und das allergische Asthma bronchiale eher als eine Entität und nicht als zwei getrennte Erkrankungen anzusehen sind. Ein Allergen führt bei einem Patienten mit entsprechender Sensibilisierung zu der gleichen allergischen Entzündungsreaktion in den Schleimhäuten der oberen wie der unteren Atemwege. Die Atopie ist neben der familiären Disposition ein wesentlicher Risikofaktor für die Entwicklung eines allergischen Asthma.
${ }^{1}$ Celedon JC, Palmer LJ, Weiss ST et al. Asthma, rhinitis, and skin test reactivity to aeroallergens in families of asthmatic subjects in Anqing, China. Am J Respir Crit Care Med 2001; 163: 1108 - 1112

2 Beasley R, Keil U, Mutius E von et al. Worldwide variation in prevalence of symptoms of asthma, allergic rhinoconjunctivitis, and atopic eczema: ISAAC. Lancet 1998; 351: 1225-1232

${ }^{3}$ Mutius E von. Determinants of childhood asthma and atopy in West and East Germany. Eur Respir J 1998; 53: 145 - 147

${ }^{4}$ Mutius E von, Fritzsch C, Weiland SK et al. Prevalence of asthma and allergic disorders among children in united Germany: a descriptive comparison. BMJ 1992; 305: 1395 - 1399

${ }^{5}$ Mutius E von, Weiland SK, Fritzsch C et al. Increasing prevalence of hay fever and atopy among children in Leipzig, East Germany. Lancet 1998; 351: $862-866$

${ }^{6}$ Upton MN, McConnachie A, McSharry C et al. Intergenerational 20 year trends in the prevalence of asthma and hay fever in adults: the Midspan family study surveys of parents and offspring. BMJ 2000; 321: 88 - 92

${ }^{7}$ Katelaris $\mathrm{CH}$. Allergic rhinitis and asthma: epidemiologica evidence for the link. Clin Exp All Rev 2003; 3: 5-8

${ }^{8}$ Guerra S, Sherrill DL, Martinez FD et al. Rhinitis as an independent risk factor for adult-onset asthma. J Allergy Clin Immunol 2002; 109: $419-425$

${ }^{9}$ Peroni DG, Piacentini GL, Alfonsi L et al. Rhinitis in pre-school, children: prevalence, association with allergic diseases and risk factors. Clin Exp Allergy 2003; 33: 1349-1354

${ }^{10}$ Aberg N. Familial occurrence of atopic disease: genetic versus environmental factors. Clin Exp Allergy 1993; 23: 829-834

${ }^{11}$ Aberg N, Sundell J, Eriksson B et al. Prevalence of allergic diseases in schoolschildren in relation to family history, upper respiratory infections, and residential characteristics. Allergy 1996; 51: 232 -237

${ }^{12}$ Leynaert B, Bousquet J, Neukirch C et al. Perennial rhinitis: an independent risk factor for asthma in nonatopic subjects: results from the European Community Respiratory Health Survey. J Allergy Clin Immunol 1999; 104: 301 - 304

${ }^{13}$ Linneberg A, Nielsen $\mathrm{NH}$, Madsen $\mathrm{F}$ et al. Secular trends of allergic asthma in Danish adults. The Copenhagen Allergy Study. Respir Med 2001; 95: 258-264

14 Linneberg A, Nielsen NN, Frolund L et al. The link between allergic rhinitis and allergic asthma: a prospective population-based study. The Copenhagen Allergy Study. Allergy 2002; 57: 1048 - 1052

${ }^{15}$ Katelaris CH, Carrozzi FM, Burke TV et al. Effects of intranasal budesonide on symptoms, quality of life, and performance in elite athletes with allergic rhinoconjunctivitis. Clin J Sport Med 2001; 12: 296-300

${ }^{16}$ Katelaris CH, Carrozzi FM, Burke TV et al. A springtime olympics demands special consideration for allergic athletes. J Allergy Clin Immunol 2000; 106: 260-266

${ }^{17}$ Lundback B. Epidemiology of rhinitis and asthma. Clin Exp Allergy 1998; $28: 3-10$

18 Yawn BP, Yunginger JW, Wollan PC et al. Allergic rhinitis in Rochester, Minesota residents with asthma: frequency and impact on health care charges. J Allergy Clin Immunol 1999; 103: 54-59

${ }^{19}$ Greisner WA, Settipane RJ, Settipane GA. Co-existence of asthma and allergic rhinitis: a 23-year follow-up study of college students. Allergy Asthma Proc 1998; 19: 185-188

20 Togias A. Rhinitis and asthma: evidence for respiratory system integration. J Allergy Clin Immunol 2003; 111: 1171 - 1183

21 Linna O, Kokkonen J, Lukin M. A 10-year prognosis for childhood allergic rhinitis. Acta Paediatr 1992; 81: 100-102

${ }^{22}$ Avial PC, Abisheganaden JA, Wong $\mathrm{H}$ et al. Effects of allergic inflammation of the nasal mucosa on the severity of rhinovirus 16 cold. J Allergy Clin Immunol 2000; 105: 923 -932

${ }^{23}$ Schramm B, Ehlken B, Quednau K et al. Cost of illness of atopic asthma and seasonal allergic rhinitis in Germany: 1-year retrospective study. Eur Respir J 2003; 21: 116-122

${ }^{24}$ Rhodes HL, Thomas P, Sporik R et al. A birth cohort study of subjects at risk of atopy. Twenty-two-year follow-up of wheeze and atopic status. Am J Respir Crit Care Med 2002; 165: 176 - 180

25 Jaakkola JJK, Jaakkola N, Piipari R et al. Pets, parental atopy, and asthma in adults. J Allergy Clin Immunol 2002; 109: $784-788$ 
${ }^{26}$ Warner JO, Naspitz CK, Cropp GJA. Third international pediatric consensus statement on the management of childhood asthma. Pediatr Pulmonol 1998; 25: 1 - 17

${ }^{27}$ Amercian Thoracic Society. Guidelines for Methacholine and exercise challenge testing-1999. Am J Respir Crit Care Med 2000; 161: $309-329$

${ }^{28}$ Laprise C, Boulet L-P. Asymptomatic airway hyperresponsiveness: a three-year follow up. Am J Respir Crit Care Med 1997; 156: 403 - 409

${ }^{29}$ Burrows BF, Martinez D, Halonen M et al. Association of asthma with serum IgE levels and skin test reactivity to allergens. N Engl J Med 1989; 320: $271-277$

${ }^{30}$ Sears MR, Burrows B, Flannery EM et al. Relation between airway responsiveness and serum IgE in children with asthma and in apparently normal children. N Engl J Med 1991; 325: 1067-1071

31 Polosa R, Holgate ST. Adenosine bronchoprovocation: a promissing marker of allergic inflammationin asthma. Thorax 1997; 52: 919-923

${ }^{32}$ Meer De G, Heederik D, Postma DS. Bronchial responsiveness to adenosine 5'-monophosphate (AMP) and methacholine differ in their relationship with airway allergy and baseline $\mathrm{FEV}_{1}$. Am J Respir Crit Care Med 2002; 165: 327-331

${ }^{33}$ Peachell PT, Columbo M, Kagey-Sobotka A et al. Adenosine potentiate mediator release from human lung must cells. Am Rev Respir Dis 1988; 138: 1143 - 1151

${ }^{34}$ Frye C, Heinrich J, Wjst M et al. Increasing prevalence of bronchial hyperresponsiveness in three selected areas in east Germany. Eur Respir J 2001; 18: 451 - 458

35 Peat JK, Toelle BG, Salome CM et al. Predictive nature of bronchial responsiveness and respiratory symptoms in a one year cohort study of Sydney schoolchildren. Eur Respir J 1993; 6: 662 - 669

${ }^{36}$ Ernst P, Ghezzo H, Becklake MR. Risk factors for bronchial hyperresponsiveness in late childhood and early adolescence. Eur Respir J 2002; 20: $635-639$

${ }^{37}$ Lommatzsch M, Virchow jun JC. Die allergische Entzündung der oberen und unteren Atemwege. Allergologie 2002; 25: 96-107

${ }^{38}$ Los H, Koppelman GH, Postma DS. The importance of genetic influences in asthma. Eur Respir J 1999; 14: 1210-1227

39 Doull UM, Lawrence S, Watson M et al. Allelic association of gene markers on chromosome $5 \mathrm{q}$ and $11 \mathrm{q}$ with atopy and bronchial hyperresponsiveness. Am J Respir Crit Care Med 1996; 153: 1280 - 1284

${ }^{40}$ Cookson WOCM, Sharp PA, Faux JA et al. Linkage between immunoglobulin $\mathrm{E}$ responses underlying asthma and rhinitis and chromosome 11q. Lancet 1989; 1: $1292-1295$

${ }^{41}$ Borish L. Genetics of allergy and asthma. Ann Allergy Asthma Immunol 1999; 82: $413-426$

${ }^{42}$ Collaborative study on the genetics of asthma (CSGA). A genome-wide search for asthma susceptibility loci in ethnically diverse populations. Nat Genet 1997; 26: 389-392

43 Ober C, Cox NJ, Abney M et al. Genome-wide search for asthma susceptivility loci in a founder population. The Collaborative Study on the Genetics of Asthma. Hum Mol Genet 1998; 7: 1393 -1398

${ }^{44}$ Eerdewegh P van, Little RD, Dupuis J et al. Association of the ADAM33 gene with asthma and bronchial hyperresponsiveness. Nature 2004; 418: $426-430$

${ }^{45}$ Powell RM, Wicks J, Holloway JW et al. The splicing and fate of ADAM33 transcripts in primary human airways fibroblasts. Am J Respir Cell Mol Biol 2004; 31: 13-21

${ }^{46}$ Cheng L, Enomoto T, Hirota T et al. Polymorphismus in ADAM33 are associated with allergic rhinitis due to Japanese cedar pollen. Clin Exp Allergy 2004; 34: 1192 - 1201

${ }^{47}$ Raby BA, Silverman EK, Kwiatkowski DJ et al. ADAM33 polymorphismus and phenotype associations in children with asthma. J Allergy Clin Immunol 2004; 113: 1071 - 1078

${ }^{48}$ Hashimoto T, Akiyama K, Kawaguchi $\mathrm{H}$ et al. Correlation of allergeninduced IL-5 and IL-13 production by peripheral blood T cells of asthma patients. Int Arch Allergy Appl Immunol 2004; 134: 7-11

${ }^{49}$ Wang IM, Lin H, Goldman SJ et al. STAT 1 is activated by IL- 4 and IL-13 in multiple cell types. Mol Immunol 2004; 41: $873-884$

50 Weidinger S, Klopp N, Wagenpfeil S et al. Association of a STAT 6 haplotype with elevated serum IgE1 population based cohort of white adults. J Med Genet 2004; 41: 658-663
${ }^{51}$ Zhu Y, Chen L, Huang Z et al. Cutting edge: IL-5 primes the Th2 cytokine-producing capacity in eosinophils through a STAT5-dependent mechanism. J Immunol 2004; 173: 2918-2922

52 Myrtek D, Knoll M, Matthiesen T et al. Expression of interleukin-13 receptor alpha 1-subunit ond peripheral blood eosinophils is regulated by cytokines. Immunology 2004; 112: $597-604$

53 Julius $\mathrm{P}$, Hochheim D, Boser K et al. Interleukin-5 receptors on human lung eosinophiles after segmental allergen challenge. Clin Exp Allergy 2004; 34: $1064-1070$

${ }^{54}$ Laitinen T, Polvi A, Rydman P et al. Characterization of a common susceptivility locus for asthma-related traits. Science 2004; 304: 300-304

${ }^{55}$ Holt PG, Macaubas C, Stumbles PA et al. The role of allergy in the development of asthma. Nature 1999; 402: B12-B17

${ }^{56}$ Johnston SL, Pattemore PK, Sanderson G et al. The relationship between upper respiratory infections and hospital admissions for asthma: a time-trend analysis. Am J Respir Crit Care Med 1996; 154 $654-660$

57 Peat JK, Tovey E, Toelle BG et al. House dust mite allergens: a major risk factor for childhood asthma in Australia. Am J Respir Crit Care Med 1996; 153: 141 - 146

${ }^{58}$ Mutius E von. Progession of allergy and asthma through childhood to adolescence. Thorax 2003; 51: S3 - S6

${ }^{59}$ Alving K, Lundberg JON, Lundberg JM et al. Nitric oxide in exhaled air: influence of inflammatory airway disease and smoking. Am J Respir Crit Care Med 1994; 149: A199

${ }^{60}$ Barnes P, Kharitonov SA. Exhaled nitric oxide: a new lung function test. Thorax 1996; 51: $233-237$

${ }^{61}$ Gillissen A, Löseke S, Hippeli S et al. Nitric oxide synthase gene expression in the bronchial epithelium. Current Pneumology 1997; 1: 20-30

62 Saleh D, Barnes PJ, Giaid A. Increased production of the potent oxidant peroxynitrite in the lungs of patients with idiopathic pulmonary fibrosis. Am J Respir Crit Care Med 1997; 155: 1763 - 1769

${ }^{63}$ Corry DB, Kheradmand F. Induction and regulation of the IgE response. Nature 1999; 402: B18-B23

${ }^{64}$ Casale TB, Bernstein IL, Busse WW et al. Use of an anti-IgE humanized monoclonal antibody in ragweed-induced allergic rhinitis. J Allergy Clin Immunol 1997; 100: 110-121

${ }^{65}$ Shields RL, Whether WR, Zioncheck K et al. Inhibition of allergic reactions with antibodies to IgE. Int Arch Allergy Immunol 1995; 107: $308-312$

${ }^{66}$ Numata T, Konno A, Yamakoshi T et al. Comparative role of peptide leukotrienes and histamine in the development of nasal mucosal swelling in nasal allergy. Ann Otol Rhinol Laryngol 1999; 108 $467-473$

67 Wang D, Clement P, Smitz J et al. Correlations between complaints, inflammatory cells and mediator concentrations in nasal secretions after nasal allergen challenge and during natural allergen exposure. Int Arch Allergy Appl Immunol 1995; 106: 278 -285

${ }^{68}$ Csoma Z, Kharitonov SA, Balint B et al. Increased leukotrienes in exhaled breath condensate in childhood asthma. Am J Respir Crit Care Med 2002; 166: 1345 - 1349

69 Pauwels RA, Brusselle GG, Tournoy KG et al. Cytokines and their receptors as therapeutic targets in asthma. Clin Exp Allergol 1998; 28: 1 -5

${ }^{70}$ Philip G, Malmstrom K, Hampel Jr SF et al. Montelukast for treating seasonal allergic rhinitis: a randomized, double-blind, placebo-controlled trial performed in the spring. Clin Exp Allergy 2002; 32: $1020-1028$

${ }^{71}$ Adelsberg J von, Philip G, LaForce CF et al. Randomized controlled trial evaluating the clinical benefit of montelukast for treating spring seasonal allergic rhinitis. Ann Allergy Asthma Immunol 2003; 90: $214-222$

72 Wilson AM, Dempsey OJ, Sims EJ et al. A comparison of topical budesonide and oral montelukast in seasonal rhinitis and asthma. Clin Exp Allergy 2001; 31: 616-624

73 Piatti G, Ceriotti L, Cavallaro G et al. Effects of zafirlukast on bronchial asthma and allergic rhinitis. Pharmacol Res 2003; 47: 541 - 547

74 Gillissen A, Bergmann K-C, Kleine-Tebbe J et al. Die Bedeutung der spezifischen Immuntherapie bei allergischem Asthma bronchiale. Dtsch. med. Wschr. 2003; 5: 204-209 